

\section{Metronidazole-Induced and Wernicke Encephalopathy: Two Different Entities Sharing the Same Metabolic Pathway?}

We read with interest the recent article by Kim et $\mathrm{al}^{1}$ on MR imaging features of metronidazole-induced encephalopathy (MIE). They described MR findings from 7 patients treated with metronidazole, 5 of whom suffered from gastrointestinal tract disease. All of the patients showed symmetric signal intensity alterations in the dentate nuclei, which is considered the main MR finding in MIE. In addition, 6 of 7 patients showed symmetric alterations in the vestibular nuclei. Alterations were also found in the midbrain, dorsal pons, medulla, splenium, and subcortical white matter. ${ }^{1}$ Interestingly, selective involvement of the vestibular nuclei $(n=6)$, abducens nuclei $(n=4)$, and red nuclei $(n=3)$ was seen. Selective involvement of cranial nerve nuclei (facial, vestibular, and abducens) and dentate nuclei has also been reported in nonalcoholic patients affected by Wernicke encephalopathy (WE). ${ }^{2,3}$ In their paper, Kim et $\mathrm{al}^{1}$ argued that, in the WE case reports mentioned above, MIE was not excludible, because both patients were on metronidazole (S.J. Bae and G. Choi, written communications, December 2006). On the contrary, there was no history of metronidazole therapy in a malnourished WE patient, ${ }^{4}$ showing MR findings similar to those described by Bae et al and Kang et $\mathrm{al}^{2,3}$ and in MIE patients. ${ }^{1}$ In MIE patients, the splenium of the corpus callosum may be also involved. ${ }^{1}$ Reversible alteration of the splenium is seen in toxic, demyelinating, infectious encephalopathies, and after seizures but also in WE. In WE, typical findings are represented by symmetric alterations in the thalami, mamillary bodies, tectal plate, and periaqueductal area. No alterations in the medial thalami and the periventricular regions of the third ventricle were seen in the presented case series ${ }^{1}$; however, all of the patients showed signal intensity alterations in the tectum or in the tegmentum, which are seen also in WE. ${ }^{4}$ We noted that, in the presented MIE patient population, the most frequent underlying pathology was gastrointestinal tract disease, which is also one of the most frequent causes of chronic or acute vitamin B1 malabsorption. Furthermore, liver cirrhosis was present in 3 of 7 patients. ${ }^{1}$ There is evidence that impaired liver function may worsen vitamin B1 deficiency in WE alcoholic patients. The mechanism of metronidazole neurotoxicity has not been completely elucidated, though its conversion to a thiamine analog and consequent vitamin B1 antagonism may play a role. On the other hand, transient edema in symmetric brain areas, especially in the posterior pons and medulla, represents a common MR finding both in MIE and in WE patients. ${ }^{1-4}$ In particular, MR imaging lesions typical of MIE closely resemble those observed in atypical nonalcoholic WE malnourished patients. Therefore, it is tempting to speculate that, in selected patients with malabsorption, MIE may be mediated by pathogenic pathway(s) similar to those thought to be operating in WE, including impairment of vitamin B1 action.

\section{References}

1. Kim E, Na DG, Kim EY, et al. MR imaging of metronidazole-induced encephalopathy: lesion distribution and diffusion-weighted imaging findings. AJNR Am J Neuroradiol 2007;28:1652-58

2. Bae SJ, Lee HK, Lee JH, et al. Wernicke's encephalopathy: atypical manifestation at MR imaging. AJNR Am J Neuroradiol 2001;22:1480-82

3. Kang SY, Kang JH, Choi JC, et al. Wernicke's encephalopathy: unusual manifestation on MRI. J Neurol 2005;252:1550-52

4. Nolli M, Barbieri A, Pinna C, et al. Wernicke's encephalopathy in a malnourished surgical patient: clinical features and magnetic resonance imaging. Acta Anaesthesiol Scand 2005;49:1566-70

$$
\begin{array}{r}
\text { G. Zuccoli } \\
\text { Department of Radiology } \\
\text { N. Pipitone } \\
\text { Department of Internal Medicine } \\
\text { Arcispedale Santa Maria Nuova } \\
\text { Reggio Emilia, Italy } \\
\text { D. Santa Cruz } \\
\text { Department of Radiology } \\
\text { Hospital Italiano }
\end{array}
$$

DOI 10.3174/ajnr.A1142 